\title{
Fortbildung
}

Schweiz Z Ganzheitsmed 2013;25:106-109

DOI: $10.1159 / 000350183$

\section{Schweizerische Jahrestagung für Phytotherapie: Veterinärmedizinischer Workshop zu pflanzlichen Antiinfektiva}

\begin{abstract}
«Pflanzliche Arzneimittel als wirksame Multi-Target-Wirkstoffe: Ein naturstoffpharmakologischer Streifzug durch die Traditionelle Europäische Medizin»
\end{abstract}

Der Workshop begann mit einem Aufruf an die Individualisierung der Medizin durch Prof. Dr. Matthias Melzig von der Freien Universität Berlin. Die Teilnehmenden erhielten einen Einblick in die Paradigmen für die Entwicklung von Antiinfektiva und zu den Personen, die dazu beigetragen haben. So hat Paul Ehrlich 1906 die Entwicklung der Chemotherapeutika, die das Prinzip der selektiven Toxizität auf den Erregermetabolismus nutzen, stark beeinflusst, während Alexander Flemming 1928 das Penicillin entdeckte und daher mit der Antibiotika-Entwicklung assoziiert wird. Können diese Effekte auch mit pflanzlichen Stoffen hervorgerufen werden? Wenn man bedenkt, dass Pflanzen seit mehr als 400 Jahren mit verschiedenen Indikationen, unter anderem auch Infektionen, eingesetzt werden, liegt es nahe, dass dies der Fall ist. Interessant ist, dass in verschiedenen Kulturkreisen ähnliche Pflanzen (unter anderem Arnikakraut, Beinwellkraut, Eichenrinde, Johanniskraut, Kamillenblüten, Ringelblume, Tormentillwurzelstock usw.) zur Behandlung von typischen Infektionen eingesetzt werden. Pflanzen sind Vielstoffgemische und Multitarget-Wirkstoffe, wodurch sich eine Breitspektrumwirkung ergibt. Es sind vor allem die phenolischen Verbindungen (z.B. in Bärentraubenblättern, Islandmoos oder Johanniskraut), die terpenoiden Verbindungen in ätherischen Ölen

Der veterinärmedizinische Workshop (Abb. 1) anlässlich der 27. Schweizerischen Jahrestagung für Phytotherapie in Baden löste dieses Jahr mit knapp 70 tierärztlichen Teilnehmern und Teilnehmerinnen aus der Schweiz, aus Deutschland, aus Österreich und aus den Niederlanden eine erfreulich gute Resonanz aus.

(z.B. in Anisfrüchten, Fenchelfrüchten oder Lavendelöl), die Senfglykoside (z.B. in Senfsamen, Kapuzinerkresse oder Brunnenkressekraut), die Alliine und Allin-Derivate (z.B. in Knoblauchzwiebeln, Küchenzwiebeln oder Bärlauchblättern), Saponine (z.B. in Efeublättern, Süssholzwurzeln oder Goldrutenkraut) und Fettsäuren (z.B. Hopfenöl, Rosenöl oder Palmfett), die hier aktiv sind, wobei keine plasmidübertragbaren Resistenzen bekannt sind. Diese Stoffe können nicht nur therapeutisch, sondern auch pro-

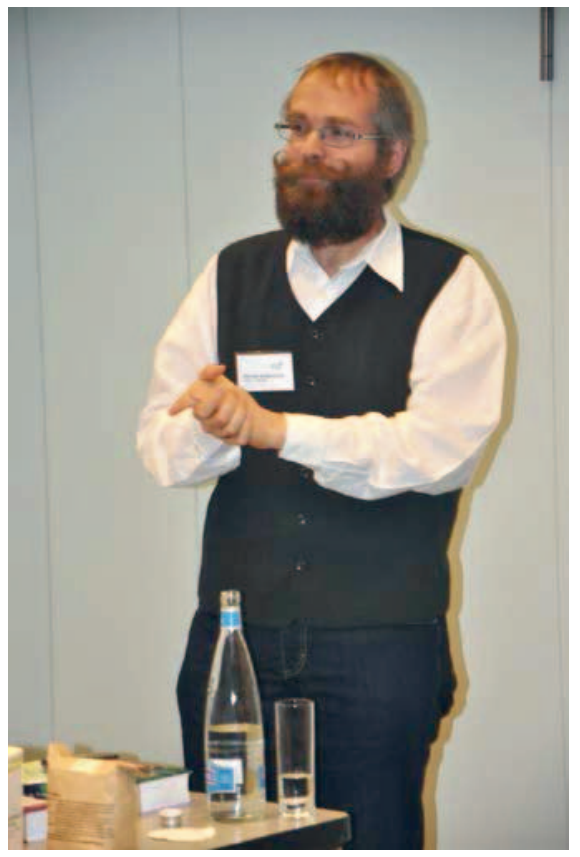

Abb. 1. Dr. med. vet. Michael Walkenhorst leitet die veterinärmedizinische Sektion der SMGP und führte durch den Workshop. phylaktisch verwendet werden - miteinander kombiniert und auch in Kombination mit Medikamenten, wobei es $\mathrm{zu}$ einer synergistischen Wirkung kommen kann.

«Immunmodulation durch Phytotherapie in der Tiermedizin»

Die Veterinärimmunologin und TCM-Tierärztin Dr. Sabine Vollstedt führte in die Immunologie ein und zeigte Möglichkeiten auf, wie mit pflanzlichen Produkten auf verschiedenen Ebenen der Immunabwehr eingegriffen werden kann. Sie gab eine kurze Übersicht über die Arten der Infektion - extrazellulär durch Viren, Bakterien, Pilze, Protozoen und Würmer oder intrazellulär im Zytoplasma (durch Viren, Chlamydien, Rickettsien, Listerien oder Protozoen) oder in Vesikeln (durch Mykobakterien, Salmonellen, Leishmanien oder Trypanosomen). Je nach Erregerart und -ort werden verschiedene Teile des Immunsystems aktiviert: Bei intrazellulären Pathogenen wie Viren oder Bakterien ist es die zelluläre Immunantwort, bestehend aus natürlichen Killerzellen (NK), zytotoxischen T-Lymphozyten (CTL) und T-Helferzellen; bei extrazellulären Pathogenen wie Bakterien, $\mathrm{Pa}$ rasiten oder Pilzen ist es die humorale Immunantwort, bestehend aus den antikörperproduzierenden B-Lymphozyten und T2-Helferzellen. Nebst
(๑) 2013 S. Karger GmbH, Freiburg
Dr. med. vet. Maya Bräm Dubé, MRCVS

Dipl. Verhaltensmedizin STVV

Cert. veterinary herbal medicine, Integrate CPD Ltd.

medvet@mayanimal.ch 
der spezifischen Immunabwehr der T-Helferzellen, zytotoxischen T-Zellen und B-Lymphozyten gibt es auch die Makrophagen und die NK, die für die unspezifische Immunabwehr verantwortlich sind. Eine weitere wichtige Funktion übernimmt die dendritische Zelle, die Erreger über spezifische Rezeptoren erkennt und je nach Erregertyp unterschiedliche Zytokine produziert: Bei extrazellulären Viren oder Bakterien wird der Tumornekrosefaktor alpha ( $\mathrm{TNF} \alpha)$ produziert, bei intrazellulären Bakterien das Interleukin-12 (IL-12), bei extrazellulären Bakterien, Parasiten und Pilzen das IL-4 und bei Viren die Interferone alpha und beta (INF $\alpha, \beta)$. Pflanzliche Wirkstoffe können auf verschiedenen Ebenen dieser Immunantworten eingreifen; so können bei viralen Infektionen z.B. der Sonnenhut, das Johanniskraut oder der Ingwer eingesetzt werden, bei einer intrazellulären bakteriellen Infektion z.B. der Grüntee, der Sonnenhut und der Knoblauch, bei extrazellulären Infektionen z.B. der Knoblauch, der Sonnenhut oder die Zimtrinde. Am Beispiel einer Lungeninfektion zeigte Sabine Vollstedt auf, an welchen Punkten gewisse Pflanzen eingreifen können. Eukalyptusöl hemmt z.B. die TNF $\alpha$-Produktion sowie die Makrophagen bei einem intrazellulären $\mathrm{Pa}$ thogen und die IL-4-Produktion bei einer extrazellulären Infektion. Teebaumöl greift ebenfalls auf der Ebene der Makrophagen ein. Bei Lungeninfektionen ist die Inhalation sinnvoll, da so die Wirkstoffe direkt an den Zielort gelangen, wobei beim Teebaumöl daran gedacht werden muss, dass es für Katzen toxisch ist. Als Teeaufguss oder Abkochung kann z.B. Isländisches Moos eingesetzt werden, das ebenfalls in intra- und extrazelluläre Prozesse eingreift; so wirkt es auf die IL-12-Produktion sowie die Makrophagen bei einem intrazellulären Pathogen und auf die IL-4- und IL-10-Produktion bei einem extrazellulären Pathogen.
«Phytotherapie als erfolgversprechende Option gegen rezidivierende und chronische Infekte bei Kleintier und Pferd" Dr. med. vet. Cäcilia Brendieck-Worm bekräftigte, dass der Antibiotika-Einsatz wichtig sei, aber dass er überlegt geschehen sollte und zwingend auf das unvermeidbare Mass beschränkt bleiben müsse. Dazu brauche es «Behandlungsoptionen bei Primärerkrankungen, die eine bakterielle Sekundärinfektion vermeiden helfen, indem sie die körpereigenen Schutz- und Regulationsmechanismen stärken», unabhängige, kritische Informationen, eine realistische Einschätzung des Risikos einer Therapie ohne konventionelle Antibiose und Konzepte zur Prävention und Nachsorge. Wichtig sei der komplementäre Einsatz der Schulmedizin mit naturheilkundlichen/regulationsmedizinischen Therapieverfahren. Die naturheilkundlichen Informationen basieren momentan noch vor allem auf der klinischen Erfahrung einzelner Tierärzte und Tierärztinnen. Hier gibt es mit Phytotherapeutika, die antientzündlich, antimikrobiell und immunstimulierend/-modulierend wirken und den ganzen Organismus unterstützen können, gute Erfahrungen im Bereich von Magen-DarmErkrankungen, Bronchitiden sowie entzündlichen Veränderungen, die primär einer antiphlogistischen Therapie bedürfen. Bei Pontus, einem 12-jährigen Warmblutwallach mit einseitigem Nasenausfluss, wurde eine Rhinitis purulenta und ein Empyem des Sinus maxillaris diagnostiziert. Das Nasensekret wurde bakteriologisch untersucht und zeigte einen hohen Gehalt an Anaerobiern (Prevotella spp.), Enterobacter agglomerans, Staphylococcus epidermidis, Pseudomonas und einen mässigen Gehalt an Aspergillus spp. auf. Ziel der Therapie war, die körpereigene Abwehr des Pferdes zu stärken, die Anzahl der Keime zu reduzieren, die Entzündung zu hemmen und die Regeneration der nasalen Schleimhäute zu unterstützen. Das Pferd wurde vom Boden gefüttert, hatte stundenweise Weidegang, inhalierte alkoholisches Thymian-Extrakt und ätherisches Kamillenöl per Druckzerstäuber (möglichst zweimal täglich), erhielt EquiMun ${ }^{\circledR} \mathrm{PLV}$ (Echinacea purpurea, Phosphorus D4, Thuja occidentalis D4) s.c. erst jeden zweiten Tag, dann zweimal wöchentlich, Mucosa comp. ad us. vet (Homöopathie) und Mucosa nasalis suis-Injeel je eine Ampulle s.c. jeden zweiten Tag und alternierend die Sinusitis-Nosode-Injeel eine Ampulle s.c. Nach 3 Wochen fand sich eine deutliche Besserung, und einen Monat nach Therapiebeginn war auch die mykologische und bakteriologische Untersuchung des $\mathrm{Na}$ sensekrets deutlich verbessert. Auf Verlangen des Tierbesitzers wurde dann eine Antibiose mit Cobactan ${ }^{\circledR}$ begonnen, woraufhin sich das Allgemeinbefinden des Pferdes verschlechterte. Die erneute Untersuchung des Nasensekrets ergab wieder Aspergillen, beta-hämolysierende Streptokokken, E. agglomerans und Serratia marcescens, worauf die Therapie umgestellt wurde auf Mucosa comp., Mucosa nasalis suis-Injeel, EquiM ${ }^{\circledR}$, Sinusitis-Nosode und Inhalation mit Thymian/Kamille; zusätzlich wurde EquiPulmin ${ }^{\circledR}$ liquid (Thymianextrakt, Efeuextrakt) verabreicht. Daraufhin erholte sich das Pferd und war nach 6 Wochen beschwerdefrei. Der zweite Fall, den Dr. Brendieck-Worm vorstellte, war die 5-jährige Eseldame Ophelie, die wegen Kopfschiefhaltung vorgestellt und ebenfalls mit einer Rhinitis purulenta und einem Empyem des Sinus maxillaris diagnostiziert wurde. Auch hier ergab die bakteriologische Untersuchung des Nasensekrets das Vorhandensein von Bakterien und Candida krusei. Ophelie wurde mit Tarantula-Iogoplex $^{\circledR}$ (Tarantula D6, Acidum silicicum D6, Hepar sulfuris D8, Pyrogeniumnosode D8), Membrana nasalium comp $^{\circledR}$ (Argentum metallicum D29 aquos, Echinacea pallida e radice ferm D2, Membrana sinuum parana- 
salium bovis D16), EquiPulmin ${ }^{\circledR}$, Inhalation mit alkoholischem Kamillenextrakt (Kamillosan ${ }^{\circledR}$ ) und Thymianöl sowie Primavera ${ }^{\circledR}$ behandelt. Zusätzlich wurden ihr lokal Kartoffelkataplasmen aufgelegt. Nach einem anfänglich vermehrten Nasenausfluss verbesserte sich die Symptomatik bis zur vollständigen Verheilung ohne Rezidiv. Sina, eine 5-jährige BorderCollie-Hündin, wurde wegen häufigem Harndrang vorgestellt und mit einer hämorrhagischen Zystitis mit Tripelphosphaten diagnostiziert. Die Therapieziele waren eine Senkung des $\mathrm{pH}-W e r t e s$, eine Steigerung der Trink- und Harnmenge, eine antimikrobielle Behandlung und eine Reduzierung der Ausgangssubstanzen für Struvit. Dies wurde angestrebt durch die Verabreichung von Cetebe ${ }^{\circledR}$ Vitamin C Retard, Canthalus Canis Oraplex ${ }^{\circledR}$ (Betula alba, Equisetum arvense, Levisticum officinalis, Urtica dioica, Acidum benzoicum), Ango$\operatorname{cin}^{\circledR}$ Anti-Infekt (Kapuzinerkressenkrautpulver, Meerrettichwurzelpulver) und eine Futterumstellung auf Hill's S/D. Schon am nächsten Tag war die Hündin beschwerdefrei; nach 14 Tagen wurde das Solidagoren ${ }^{\circledR}$ liquid (Goldrutenkraut, Gänsefingerkraut, Schachtelhalmkraut) hinzugefügt, nach dem 30 . Behandlungstag ist die Urinuntersuchung ohne besonderen Befund und es tritt kein Rezidiv mehr auf.

\section{«Phytotherapeutische Behandlung von Infektionskrankheiten beim Nutztier»}

Dr. med. vet. Elisabeth Stöger (Abb. 2) aus Österreich fokussierte auf Durchfallerkrankungen und mögliche Behandlungsarten mit Phytotherapeutika bei Nutztieren. Sie präsentierte in einem gut strukturierten, breitgefächerten Vortrag anhand von den verschiedenen Wirkungsmechanismen, die bei Durchfallerkrankungen wichtig sind, die entsprechenden Wirkstoffe von relevanten Pflanzen, die bei dieser Symptomatik eingesetzt

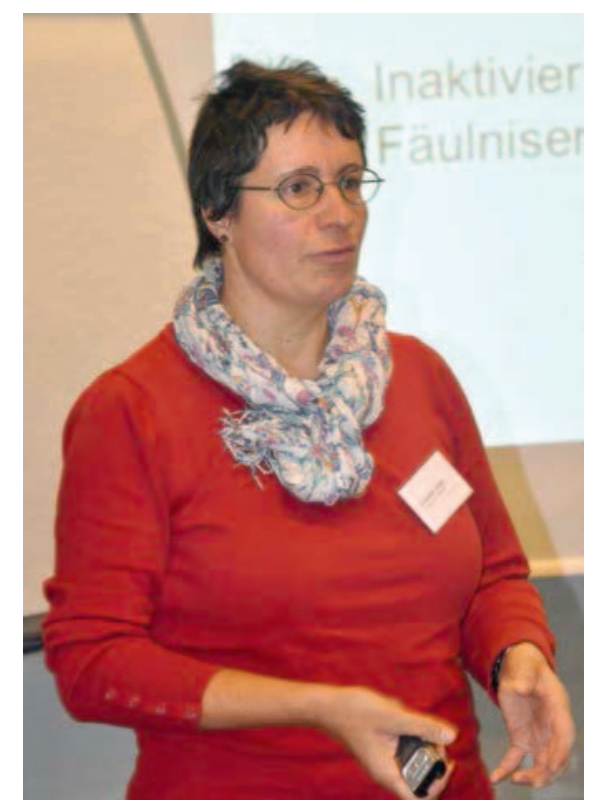

Abb. 2. Dr. med. vet. Elisabeth Stöger aus Feldkirchen in Kärnten ist eine der wenigen Tierärztinnen im europäischen Raum, die sich eine umfassende Erfahrung beim Einsatz von pflanzlichen Arzneimitteln bewahrt haben.

werden, und mögliche Zubereitungsarten. So wirken z.B. in der Eichenrinde, im Blutwurz, im Schwarztee, in Fichtennadelspitzen, in getrockneten Heidelbeeren und in Hamamelis vorhandene Gerbstoffe adstringierend, entzündungshemmend, reizmildernd, lokalanästhetisch, styptisch, sekretmindernd und karminativ. Im Detail ist Elisabeth Stöger auf die Eichenrinde eingegangen - hier wird die Rinde der jüngeren Zweige als Dekokt (Abkochung) oder Pulver bei Durchfallerkrankungen eingesetzt. Äusserlich kann die Eichenrinde bei Euter-Schenkel-Ekzem, Nekrosen und alten Wunden, entzündlichen Hauterkrankungen sowie «Hot Spots» helfen. Auch Schwarztee gehört zu dieser Kategorie und kann z.B. als Dekokt bei der infektiösen Keratokonjunktivitis der Schafe Abhilfe schaffen. Als Fertigarzneimittel existiert das Stullmisan-Pulver ${ }^{\circledR}$ aus Fichtennadelspitzen auf dem Markt. Die zweite vorgestellte Wirkstoffgruppe waren die Ätherisch-Öl-Drogen, d.h. leicht flüchtige, lipophile Stoffe, die durch Destillation gewonnen werden und eine antimikrobielle, sekretions- fördernde, spasmolytische, sekretolytische, appetitanregende, verdauungsfördernde, karminative, gärungshemmende und - topisch eingesetzt - repellierende Wirkung haben. $\mathrm{Zu}$ dieser Gruppe gehören unter anderem Kamille, Schafgarbe, Ringelblume, Thymian, Salbei, Pfefferminze, Eukalyptus und der Kampferbaum. Als Fertigarzneimittel ist z.B. die Eucacomp-Suspension ${ }^{\circledR}$ - bestehend aus Eukalyptusöl, Ringelblume, Majoran und Melisse - zur intrauterinen Behandlung von Endometritiden sowie Sterilität und zur Scheiden- und Zervixspülung auf dem Markt. In dieser Kategorie der Ätherisch-Öl-Drogen wurde speziell die Kamille (Matricaria recutita) als Teezubereitung (Aufguss) und eine bei Kälbern einsetzbare Teemischung aus Kamille, Pfefferminze und Schafgarbe hervorgehoben.

«Einsatz pflanzlicher Hausmittel in der Schweiz bei Wunden und Hautinfektionen von Nutztieren» Zurück zum Ursprung der Phytotherapie in der Schweiz ging es schlussendlich im letzten Vortrag, in dem die Pharmaziestudentin Monika Disler (Abb. 3) ihre ethnoveterinärmedizinische Studie betreffend des Einsatzes von Pflanzen bei Wunden und Hautveränderungen durch Landwirte in den Kantonen Aargau, Appenzell Ausserrhoden und Innerrhoden, Schaffhausen, St. Gallen, Thurgau und Zürich vorstellte. Sie hatte zusammen mit der Pharmaziestudentin Kathrin Schmid in ihrer Studie untersucht, welche Präparate mit Anwendung auf der Haut und mit dem Indikationsgebiet Haut im Wissensursprung der Vorfahren/Familie der heutigen Bauern von diesen mehr als fünfmal eingesetzt worden sind. Insgesamt wurden 34 Rezepte genannt, die diese Bedingungen erfüllten; für diese wurden 49 Anwendungen zur Versorgung von Wunden und Entzündungen oder anderen Veränderungen der Haut beim Tier 
beschrieben. Die Anwendungen umfassten das Auftragen auf die Haut, Kompressen/Verbände/Wickel, Bäder und Auswaschungen/Spülungen. Die von den Landwirten meistgenannten Pflanzen waren Calendula officinalis, $M$. recutita und das Johanniskraut, die am häufigsten als Extraktionen mit Öl und Fett angewendet werden, aber auch als Wasser- oder Alkoholextraktion und direkt als Frischpflanze auf die Haut appliziert wurden. Speziell eingegangen ist Monika Disler auf den Wallwurz-Umschlag bei entzündeten Sprunggelenken, den Kamillentee zur Auswaschung von entzündeten Wunden, die Ringelblumentinktur zur Auswaschung von Wunden und eine Harzsalbe zur Wundbehandlung. Um die Rezepturen vergleichen zu können, musste die Konzentration der wirksamen Pflanze auf Gramm Drogenäquivalent pro $100 \mathrm{~g}$ Endprodukt umgerech-

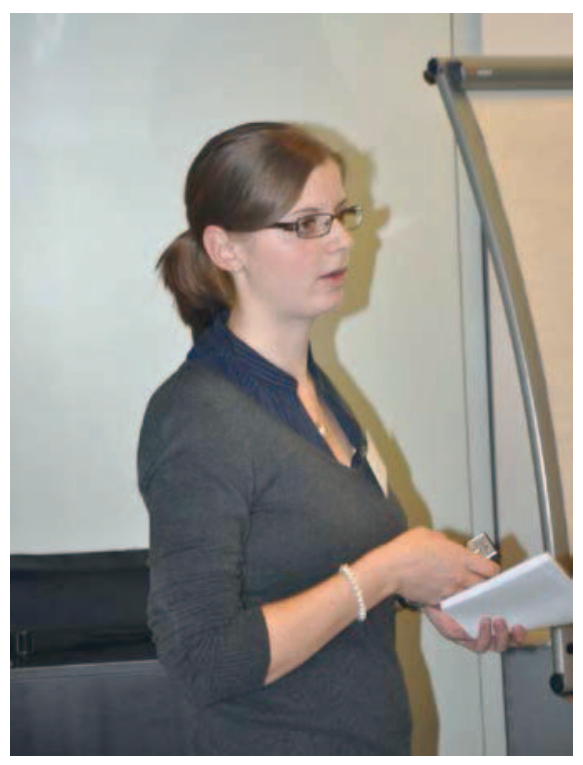

Abb. 3. Feldforschung: Die Pharmaziestudentin Monika Disler berichtete von ihrer Feldforschung zum Thema Hausmittel. Sie legte den Fokus auf Wunden und Hautveränderungen. net werden. Eine wichtige Indikation war die Rinderflechte, wofür $25 \mathrm{Re}$ zepte gefunden wurden. Dies ließ sich unterteilen in 19 Umgebungsbehandlungen, z.B. das Aufhängen von Schwarzdorn, Weissdorn, Kreuzdorn oder Stechpalme im Stall, und 6 lokale Behandlungen der veränderten Hautstellen mit Lavendelöl, Rapsöl oder anderem Speiseöl, Salbeitinktur oder Storchenschnabelsalbe. Weitere ähnliche Arbeiten in anderen Gebieten der Schweiz sind geplant.

\section{Kontakt}

Geschäftsstelle SMGP

c/o Zürcher Hochschule für angewandte Wissenschaften

Grüental/Postfach, 8820 Wädenswil, Schweiz

\section{SMGP-vet}

Dr. med. vet. Michael Walkenhorst

Forschungsinstitut für biologischen Landbau FiBL Ackerstrasse 21, Postfach, 5070 Frick, Schweiz

\section{Fort- und Weiterbildung in Veterinärphytotherapie}

Nach den Ärzten und den Apothekern haben nun auch die Tierärzte einen Weiterbildungstitel in Phytotherapie: Dieser wurde Ende 2012 in Zusammenarbeit mit der Schweizerischen Tierärztlichen Vereinigung für Komplementär- und Alternativmedizin (www.camvet.ch) geschaffen. Der neue Fähigkeitsausweis Veterinärphytotherapie GST basiert auf der Weiterbildung, die die Schweizerische Medizinische Gesellschaft für Phytotherapie (SMGP) seit vielen Jahren mit Erfolg anbietet. Die SMGP garantiert Kurse auf wissenschaftlicher Basis mit Hochschulniveau. Das sorgt für spannende Diskussionen. Neben einem allgemeinen Teil für alle vertretenen Berufsgruppen (Humanmediziner, Veterinärmediziner, Pharmazeuten) bietet die SMGP an allen Kursen einen veterinärspezifischen Block an. Fallbeispiele und praktische Tipps zur Anwendung werden aufgezeigt und zu den einzelnen Arzneipflanzen Profile erarbeitet, die aus einem pharmazeutischen und einem veterinärspezifischen Teil bestehen. Der Bezug zur Natur geht dabei nicht vergessen: Auf Exkursionen werden die Pflanzen an ihrem natürlichen Standort betrachtet. Details zur Weiterbildung mit den Terminen und die entsprechenden Reglemente finden Sie jederzeit aktuell auf www.smgp.ch. Der Zyklus ist modular aufgebaut und ein Einstieg deshalb jederzeit möglich. Ideal für den Start ist der Kurs 1, der jeweils von Donnerstag bis Samstag in Engelberg stattfindet. 2013 wird dieser Anlass vom 23. bis 25. Mai angeboten. Es sind noch Plätze frei.

Akkreditierung: Bildungspunkte für VeterinärmedizinerInnen:

- GST: 2 Punkte pro Kurstag, Exkursionstag und Tagung, 5 Punkte Kurs 1.

- ATF: 5 Stunden pro Kurstag, 19 Stunden für Kurs 1. 